\title{
Validación del Índice de Bien-estar Personal en personas con enfermedad renal crónica
}

\author{
Luís Manuel Mota de Sousa ${ }^{1,2}$, Cristina María Alves Marques-Vieira3 ${ }^{3}$, Sandy Silva Pedro Severinoㄹ, Juan \\ Luis Pozo Rosado ${ }^{4}$, Helena María Guerreiro José 5 \\ ${ }^{1}$ Hospital Curry Cabral del Centro Hospitalar Lisboa Central. Lisboa. Portugal \\ ${ }^{2}$ Universidad New Atlântica. Oeiras. Portugal \\ ${ }^{3}$ Instituto de Ciências de la Salúd, Universidad Católica Portuguesa. Lisboa. Portugal \\ ${ }^{4}$ Hospital Professor Doutor Fernando Fonseca. Lisboa. Portugal \\ ${ }^{5}$ Escuela de Salud. Multipefil. Luanda. Angola
}

\section{Resumen}

Objetivo: verificar las propiedades psicométricas del Índice de bien-estar personal (IBP) en personas con enfermedad renal crónica en programa de hemodiálisis.

Método: Se trata de un estudio metodológico. La muestra aleatoria es constituida por 171 personas con Enfermedad Renal Crónica (ERC) sometida a hemodiálisis en dos clínicas en la región de Lisboa, Portugal. La recogida de datos fue realizada entre mayo y junio de 2015. Se evaluaron las propiedades psicométricas: validad (constructo, predictiva convergente, y discriminativa), confiabilidad por medio de la consistencia interna ( $\alpha$ de Cronbach) y estabilidad (Coeficiente de Correlación de Spearman-Brown y Coeficiente de Correlación Intraclasse (CCI)).

Resultados: EI IBP presenta una dimensión ajustada a los datos. La satisfacción con la salud es lo que más contribuye para el IBP. Tiene validad convergente con Felicidad Subjetiva y Afecto Positivo y Afecto Negativo. Tiene poder discriminativo en la nacionalidad. EI $\alpha$ Cronbach fue de 0,82. Teste Re-teste para cuestionario 0,92 e CCI $=0,95$, para entrevista 0,85 y CCI= 0,89 .

Conclusiones: La versión portuguesa del IBP es válida y reproducible en personas con ERC, tanto por cuestionario como por entrevista.

\section{Correspondencia: \\ Luís Manuel Mota de Sousa \\ Fábrica da Pólvora de Barcarena, \\ 2730-036 Barcarena. Oeiras. Portugal \\ E-mail: luismmsousa@gmail.com}

PALABRAS CLAVE

- INSUFICIENCIA RENAL CRÓNICA

- DIÁLISIS RENAL

- CALIDAD DE VIDA

- LOS ESTUDIOS DE VALIDACIÓN

- PSICOMETRÍA

Validation of the Personal Wellbeing Index in people with chronic kidney disease

\begin{abstract}
Objective: to verify the psychometric properties of the Personal Wellbeing Index (PWI) in people with chronic kidney disease on hemodialysis.
\end{abstract}

Method: This is a methodological study. A random sample consisted of 171 people with Chronic Kidney Disease (CKD) who underwent hemodialysis in two clinics in the region of Lisbon, Portugal. Data collection was conducted from May to June 2015. The psychometric properties were evaluated: Validity (construct, convergent predictive and discriminative), reliability through internal consistency (Cronbach $\alpha$ ) and stability (Spearman-Brown correlation coefficient and intraclass correlation coefficient (ICC)).

Results: PWI has a dimension adjusted to the data set. Satisfaction with health is the largest contributor to the PWI. It shows convergent validity with Subjective Happiness and Positive Affect and Negative Affect. It has discriminative power of nationality. The Cronbach $\alpha$ was 0,82 . Retest test for the questionnai- 
re 0,92 and $\operatorname{ICC}=0,95$, for the interview 0,85 and ICC $=0,89$.

Conclusions: The Portuguese version of PWI is valid and reproducible in people with CKD, both by questionnaire and by interview.

\section{KEYWORDS}

- RENAL INSUFFICIENCY CHRONIC

- RENAL DIALYSIS

- QUALITY OF LIFE

- VALIDATION STUDIES

- PSYCHOMETRICS

\section{Introducción}

La Enfermedad Renal Crónica (ERC) se caracteriza por la pérdida lenta, progresiva e irreversible de la función renal, evidenciada por el aumento de los niveles séricos de urea y creatinina en la sangre y tiene como principales causas la hipertensión arterial, diabetes mellitus y glomerulonefritis ${ }^{1,2}$. La hemodiálisis (HD) es un tratamiento utilizado en personas con ERC, además es un procedimiento muy complejo y exigente que se puede tornar muy restrictivo e implicar profundas alteraciones en el estilo de vida. ${ }^{3}$ Estos cambios habitualmente pueden estar relacionados con el uso continuo de fármacos, el enfrentamiento a la dependencia de otras personas y de aparatos y de la dependencia financiera ${ }^{4}$. Además de esto, la hemodiálisis interfiere en la vida de la persona, a nivel del bien-estar y en el grado de satisfacción de la persona con ERC. ${ }^{5}$

El bien-estar se puede definir como una evaluación emocional y cognitiva en la vida de las personas y puede estar relacionado con el estado de contento, feliz, sano y próspero $^{6,7}$. Las medidas del bien-estar Subjetivo (BES) están altamente reconocidas por tener una aplicación útil en la evaluación de la calidad global y sus componentes ${ }^{6}$. El Indice de Bien-estar Personal/Satisfacción con la vida en general (IBP/SVG) es un cuestionario que permite evaluar el BES. Fue desarrollado en International Wellbeing Group para evaluar el BES a partir de la satisfacción con dominios específicos de vida. ${ }^{7}$ EI IBP (PWI de The Personal Wellbeing Índex en el original) tuvo por base la Comprehensive Quality of Life Scale ${ }^{8}$. EI IBP con 7 dominios está validado en varias países, como Portugal $^{7}$, Brasil, Colombia y Canadá ${ }^{\text {, Austrália }}{ }^{10}$, China $^{11-12}$, Tailandia ${ }^{13}$, Nueva Zelandia (descendiente euro- peos y maorí $)^{14}$ y España ${ }^{15}$. En el análisis factorial utilizan el análisis factorial exploratorio, método de Análisis de Componentes Principales y rotación varimax ${ }^{7,9-12,14}$, análisis factorial confirmatória ${ }^{7}$ y análisis de Rasch. La confiabilidad interna fue evaluada por la $\alpha$ de Cronbach ${ }^{7}$, 9-15, y la estabilidad por el Teste Re-teste y Coeficiente de Correlación Inter-Clases (CCI ${ }^{10}$. En la validad concurrente presentó correlaciones moderadas con el soporte social, depresión y sentido de coherencia ${ }^{15}$. En todas las culturas demostró propiedades psicométricas adecuadas, siendo considerada una medida válida y fiable.

EI BES ha ganado importancia en la investigación en el contexto social y de salud, pero ha sido estudiado en las personas con enfermedad renal en la fase final. En un estudio reciente se verifico que las personas con ERC en hemodiálisis refirieron menor satisfacción con la vida en general, en la realización personal, relacionamiento y seguridad personal en comparación con la población en general ${ }^{16}$.

En este sentido, con este estudio queremos verificar la versión portuguesa del IBP/SVG ${ }^{7}$ mantiene las propiedades psicométricas de validad y de reproductibilidad en personas con ERC en programa de hemodiálisis. Además de esto, era importante verificar el BES medido por el IBP/SVG ${ }^{7}$, relacionado a otras medidas de Bien-estar Psicológico, específicamente, el Afecto Positivo (AP) y el Afecto Negativo (AN $)^{17}$, la Felicidad Subjetiva ${ }^{18}$. Como se encontraron diferencias culturales ${ }^{9}$, queremos entender si el IBP/SVG consigue discriminar por la nacionalidad.

El objetivo de esta investigación es verificar las propiedades psicométricas del IBP/SVG en personas con ERC.

\section{Material y método}

Es un estudio metodológico ${ }^{19}$, realizado en la Clínica Diaverum, dos unidades de diálisis de la región de Lisboa, entre mayo y junio de 2015.

La población está compuesta por personas con ERC sometidas a hemodiálisis. Los criterios de inclusión utilizados fueron: personas con ERC, sujetas a tratamiento de hemodiálisis hace por lo menos seis meses, con edades igual o superior a 18 años y que consintieron libre y esclarecidamente a participar en el estudio. Los criterios de exclusión definidos fueron los siguientes: personas con déficit cognitivo y enfermedad psiquiátrica activa. En el total 253 personas con ERC cumplían los criterios de inclusión (139 en la Clínica 1 y 114 en 
la Clínica 2). La muestra fue formada por las personas que cumplían los criterios de elegibilidad y la selección fue de forma probabilística (aleatoria sin reposición, teniendo en cuenta los criterios de elegibilidad). EI cálculo de la muestra con Intervalo de Confianza (IC) de $95 \%$ y error de muestra de $5 \%{ }^{20}$, indicó una muestra necesaria de 192 personas (103 en clínica 1 y 89 en la clínica 2). Se realizó una selección aleatoria sin reposición. De los sujetos seleccionados en la Clínica 1, seis personas rechazaron participar, dos fueron ingresadas y dos desistieron, en la Clínica 2, cuatro personas rechazaron participar, dos fueron internadas y cinco desistieron. Al final se obtuvieron datos de 171 personas, 93 de la Clínica 1 (89\%) y 78(88\%) de la Clínica 2.

Para la recogida de datos se utilizaron los siguientes instrumentos: bien-estar subjetivo obtenido por el (IBP/ SVG $)^{7}, A P$ y AN medidos por la versión portuguesa de la Positive and Negative Affect Schedule (PANAS) ${ }^{17}$, felicidad por la versión portuguesa de la Subjective Happiness Scale (SHS) ${ }^{18}$ y un instrumento para caracterización del perfil de la muestra a nivel socio demográfico y clínico (edad, género, nacionalidad, escolaridad, actividad profesional, estado civil, tiempo de diálisis, presencia de hipertensión arterial y de diabetes). EI IBP está constituido por siete ítems/dominios (satisfacción con el nivel de vida, salud, realización personal, relaciones personales, sentimiento de seguridad, unión a la comunidad, y seguridad con el futuro) que pretenden evaluar la "satisfacción con la vida en general". Para cada ítem es pedido a los respondientes para indicar el grado de satisfacción en cada ítem/dominio en una escala de " 0 " (extremamente insatisfecho) a "10" (extremamente satisfecho), en que " 5 " es la posición intermedia neutra. El IBP se calcula en una nota de 0-100 (porcentaje máxima de escala (\%ME)). El análisis factorial confirmatoria de la versión portuguesa demuestra la existencia de un único factor, con una fiabilidad interna con el valor del $\alpha$ de Cronbach de $0.81^{7}$.

La PANAS se compone por dos sub escalas: AP y AN, con 10 ítems cada, en que los constructos son evaluados en una escala tipo Likert de 1 a 5. En la dimensión $A P$, ésta está más presente cuanto mayor sea la puntuación, en un máximo de 50 puntos. Lo mismo para la dimensión AN. Las cualidades psicométricas de las PANAS, en la versión portuguesa, revelan tal como la escala original, la existencia de 2 factores, una consistencia interna con el valor de la $\alpha$ de Cronbach de 0.86 (en la original, $\alpha=0.88$ ) para la escala de AP y de 0.89 (en la original, $\alpha=0.87$ ) para la escala de $\mathrm{AN}^{17}$. La SHS está constituida por cuatro ítems, en que en dos afirmaciones se pide a los respondientes para que se caractericen a si propios por comparación con los otros, tanto en condiciones absolutas como relativas (ítems dos y tres), y otros dos ítems corresponden a descripciones de felicidad y infelicidad. En esta escala se pide a los participantes para indicar la extensión en que las afirmaciones los caracterizan, con la respuesta dada en una escala visual analógica con siete puntos, fundamentadas en dos afirmaciones antagónicas que expresan el nivel de felicidad o su falta. La versión portuguesa presenta un único factor con una Confiabilidad Interna con el valor del $\alpha$ de Cronbach de $0,76^{18}$.

Los análisis estadísticos se realizaron con el Statistical Package for Social Sciences (SPSS) versión 20.0. En la evaluación de las propiedades psicométricas, el estudio de la confiabilidad, se efectuó por medio del $\alpha$ de Cronbach y para evaluar la estabilidad se usó el CCI y Coeficiente de Correlación de Spearman-Brown ${ }^{21}$ en el Teste Re-teste (después de 48 a 96 horas en 40 personas seleccionadas aleatoriamente, 26 por auto relleno y 14 por entrevista). Se adoptó un valor mínimo de 0,70 como una Consistencia Interna satisfatória ${ }^{21}$. En el estudio de la validad, el Análisis Factorial Exploratorio (AFE) fue por el método de la máxima verosimilitud, con rotación Variamax. La adecuación fue evaluada por el Kaiser-Meyer-Olkin (KMO) y teste de esfericidad de Bartlett. La validad convergente se evaluó a través de la correlación de Pearson entre el IBP la PANAS, la SHS. Para verificar la validad predictiva de las dimensiones de la IBP se efectuó un análisis de regresión múltiple jerárquica. La estadística $\mathrm{sr}^{2}$ representa la proporción de variancia única que es la contribución de cada ítem/ dominio. El es calculado como el cuadrado de la estadística 'part' retirada del análisis de regresión múltiple. Este valor multiplicado por 100 da el porcentaje de variancia única con que ese ítem contribuye para el IBP/SVG. Para verificar la validad discriminante se uso el Teste $t$ Student para muestras independientes. Las variables categóricas se expresaron como porcentual o valor absoluto; las continuas como medias \pm desvío estándar o mediana. El nivel de significancia adoptado fue de $p<0,05$.

Se pidió la autorización de las versiones portuguesas do $\mathrm{IBP}^{7}$, de la PANAS $^{17}$ y de la $\mathrm{SHS}^{18}$, y se concedió.

Este estudio se aprobó por la Comisión de Ética de la Diaverum ( $\left.{ }^{\circ} 1 / 2015\right)$. Todos los participantes firmaron el plazo de consentimiento libre y esclarecido después de haber sido informados sobre la garantía de confidencialidad de sus datos y del derecho de renuncia, sin cualquier tipo de perjuicio. 


\section{Resultados}

El perfil socio demográfico y de salud de los participantes:

La media de edad de la muestra fue de 60,20 $\pm 14,34$ años, constituida mayoritariamente por hombres $(61 \%)$. En relación a la nacionalidad, la mayoría eran de nacionalidad portuguesa $(80,1 \%), 14 \%$ cabo verdiana, 3,5\% santomense, $1,8 \%$ angolana y 0,6 guíenense. En la escolaridad, $3,6 \%$ de analfabetos, $42,9 \%$ en el $4^{\circ}$ año, $18,5 \%$ con el $6^{\circ}$ año, $14,9 \%$ con el $9^{\circ}$ año, $11,3 \%$ con el $12^{\circ}$ año y $8,9 \%$ con educación superior. EI $25,9 \%$ de los sujetos eran solteros, $56,5 \%$ casados, $11,8 \%$ viudos y 5,9 divorciados. En lo que se refiere a la actividad laboral $76,7 \%$ estaban jubilados y $23,3 \%$ tenían una actividad profesional regular. A nivel de los datos de salud, los sujetos de la muestra recibían hemodiálisis desde hace $72,17 \pm 54,23$ meses, $62,1 \%$ tenían hipertensión arterial y el 27,1\% diabetes.

En el análisis de las propiedades psicométricas del IBP, comprobadas a través del coeficiente de $\alpha$ de Cronbach, varió de 0,78 a 0,84, después de la exclusión de cada uno de los ítems.

En el estudio de la estabilidad (teste, re-teste), datos obtenidos por cuestionario $(n=26)$, tienen en una primera evaluación un $\alpha$ de Cronbach global de 0,89 y en una segunda evaluación de 0,94. El Coeficiente de Correlación de Spearman-Brown fue de 0,92 y el CCI fue de 0,95 [IC95\%; 0,92 a 0,98, $p<0,001]$. Por entrevista $(n=14)$ el $\alpha$ de Cronbach global en la primera evaluación fue de 0,76 y segunda evaluación fue de 0,86. El Coeficiente de Correlación de Spearman-Brown fue de 0,85 y el CCI fue de 0,89 [IC95\%; 0,80 a 0,96, $p<0,001$ ], lo que demuestra que hubo buena consistencia y estabilidad entre evaluaciones tanto por medio de instrumento de medida auto reportado como por entrevista.

La AFE (KMO $=0,85$; teste de esfericidad de Bartlett $\chi^{2}$ [21] 407,324, $\left.p<0,001\right)$ presentó una solución de un solo factor, que fue responsable por $50,6 \%$ de la varianza explicada del constructo. Todos los ítems se colocaron en factores, con cargas factoriales adecuadas (o sea, $>0,4$, ver Tabla 1 ). Las comunalidades $\left(h^{2}\right)$ variaron entre 0,17 e 0,59. El coeficiente $\alpha$ de Cronbach de la escala global fue de 0,82.
Tabla 1. Análisis factorial exploratorio del IBP en personas con ERC, Lisboa, Portugal, 2015.

\begin{tabular}{|l|c|c|}
\hline Dimensiones del IBP & $\begin{array}{r}\text { Factor } 1 \\
\text { IBP/SVG }\end{array}$ & $h^{2}$ \\
\hline 1. Nivel de vida & 0,77 & 0,59 \\
\hline 2. Con su salud & 0,41 & 0,17 \\
\hline 3. Conseguir obtener de la vida con el esfuerzo & 0,73 & 0,53 \\
\hline 4. Con relaciones personales & 0,69 & 0,48 \\
\hline 5. Con su seguridad & 0,63 & 0,39 \\
\hline 6. Con el sentimiento de pertenecer a la comunidad & 0,56 & 0,32 \\
\hline 7. Con la seguridad de su futuro & 0,74 & 0,55 \\
\hline Números propios (Eigenvalue) & 3,547 & \\
\hline Varianza explicada & $50,6 \%$ & \\
\hline Coeficiente de Cronbach & 0,93 & \\
\hline Media (DP) & $64,2( \pm 17,9)$ & \\
\hline
\end{tabular}

Legenda: $h^{2}$ - comunalidades. IBP/SVG- Índice de bien-estar personal /

Satisfacción con la vida en general.
Los resultados de la Tabla 2 muestran que los siete dominios del BES predicen $98,5 \%$ de la varianza de la SVG. La suma de la magnitud de varianza única para la solución en que, en este caso, es de $91 \%$ mientras que la varianza compartida por todos los ítems/dominios es de 7,5\%. El análisis de la contribución única de cada ítem/dimensión muestra que la satisfacción con la salud que contribuye con $21 \%$ para la varianza única del total de $98,5 \%$ de la varianza explicada. Siguen los dominios/ítems nivel de vida, seguridad, enlace a una comunidad, seguridad en el futuro y por fin a la realización y relaciones personales.

En el estudio de la validad convergente, se confirmó que la IBP/SVG presentó correlaciones positivas moderadas con el AP y Felicidad Subjetiva, y una correlación negativa baja con los AN. Además, se confirmó que la Felicidad Subjetiva presenta una correlación moderada con el AP (Tabla 3).

En relación a la validad discriminativa, las personas con nacionalidad de un país africano (Cabo-Verdianos, Santomenses, Angolanos y Guíenense) tienen IBP/SGV superiores a las personas de nacionalidad portuguesa $(70,47 \pm 15,9$ versus $63,15 \pm 18,3 ; \mathrm{t}$ $[168]=2,114 ; p<0,05)$. La cultura influencia IBP/ $S V G$ en las personas con ERC. 
Tabla 2. Regresión de los ítems/dominios de satisfacción con la SVG y correlaciones inter ítems, en personas con DRC, Lisboa, Portugal, 2015.

\begin{tabular}{|c|c|c|c|c|c|c|c|c|c|c|}
\hline & SGV & NV & $\mathbf{S}$ & $\mathbf{R}$ & $\mathbf{R P}$ & $\mathbf{S}$ & EC & B & B & $s \mathbf{r}^{2}$ \\
\hline NV & $0,79 *$ & & & & & & & $1,688^{*}$ & 0,25 & 0,17 \\
\hline $\mathbf{S}$ & $0,55^{*}$ & 0,31 * & & & & & & $1,452^{*}$ & 0,23 & 0,21 \\
\hline $\mathbf{R}$ & $0,75^{*}$ & $0,62 *$ & $0,32 *$ & & & & & $1,448^{*}$ & 0,19 & 0,13 \\
\hline RP & $0,72^{*}$ & $0,49 *$ & $0,25^{*}$ & $0,53^{*}$ & & & & $1,352^{*}$ & 0,17 & 0,13 \\
\hline$S$ & 0,69 * & $0,45^{*}$ & 0,26 * & $0,33^{*}$ & $0,47^{*}$ & & & $1,377^{*}$ & 0,20 & 0,16 \\
\hline EC & $0,62 *$ & $0,38^{*}$ & 0,14 & 0,49 * & 0,47 * & 0,39 * & & $1,395^{*}$ & 0,17 & 0,14 \\
\hline SF & $0,78^{*}$ & 0,60 * & 0,36 * & 0,49 * & 0,49 * & $0,56^{*}$ & 3,41 * & $1,313^{*}$ & 0,20 & 0,14 \\
\hline \multicolumn{8}{|c|}{${ }^{*} p<0,001$} & \multicolumn{2}{|l|}{$\mathrm{R}^{2}$} & 0,992 \\
\hline \multicolumn{8}{|c|}{ la Varianza única $=0,91 ;$ varianza compartida $=0,075$} & \multicolumn{2}{|l|}{ Adjusted $\mathrm{R}^{2}$} & 0,985 \\
\hline
\end{tabular}

Significado: IBP/SVG- Índice de bien-estar personal /Satisfacción con la vida en general, NV- Nivel de vida, S- Salud, R- Realización, RP- Relaciones personales, S- Seguridad, EC- Enlace a la comunidad y SF- seguridad en el futuro.

Tabla 3. Validad Concurrente y confiabilidad de las medidas. Lisboa, Portugal, 2015.

\begin{tabular}{lllll}
\hline Alfa Cronbach $(\boldsymbol{\alpha})$ & SGV/IBP & AP & AN & FS \\
SGV/IBP & $(0,82)$ & & & \\
AP & $0,33^{* * *}$ & $(0,86)$ & & \\
AN & $-0,16 *$ & 0,09 & $(0,88)$ & \\
FS & $0,61 * * *$ & $0,41 * * *$ & $-0,13$ & $(0,90)$ \\
\hline
\end{tabular}

Significado: ${ }^{*}<0,05 ;{ }^{*} p<0,01 ; * * * p<0,001 ;$ IBP/SVG- Índice de bien-estar personal /Satisfacción con la vida en general, AP- afectividad positiva, ANafectividad negativa, FS- felicidad subjetiva.

\section{Discusión}

La versión portuguesa del instrumento IBP/SVG tiene propiedades psicométricas buenas en personas con ERC. Esta medida de BES en esta población específica es confiable y reproductible. Los valores de la confiabilidad son semejantes a la versión portuguesa $(\alpha=0,81)^{7}$, la muestra de Canadá $(\alpha=0,82)^{9}$, China $(\alpha=0,80)^{11}$, Nueva Zelanda (descendientes europeos $\alpha=0,83$; descendientes maorí $\alpha=0,85)^{14}$ y España $(\alpha=0,85)^{15}$. Fue superior a las muestras de Brasil y Colombia (respetivamente, $\alpha=0,73$ e $\alpha=0,76)^{9}$, Australia $(\alpha=0,76)^{10}$ y a una otra muestra de China $(\alpha=0,71){ }^{12}$ En relación a la estabilidad, esta escala es reproductible con las dos modalidades de recogida de datos (cuestionario y entrevista). En el estudio realizado en Australia con una muestra aleatoria de 31 sujetos, el Teste Re-teste fue de 0,58 y el CCI fue de $0,57^{10}$. Todos los ítems se colocaron en un único factor, con cargas factoriales superiores a 0,41 . Confirma los resultados obtenidos en otras culturas excepto en la muestra australiana que revelo una estructura factorial con dos dimensiones (factor 1 - relacionamiento personal, seguridad en el futuro, salud y enlace a la comunidad, el factor 2 agrupo la realización, seguridad personal y nivel de vida) ${ }^{10}$. La varianza explicada del factor presenta un valor superior a $50 \%$, semejante a la muestra australiana $(57.97 \%)^{10}$. En algunas muestras la varianza fue inferior a $50 \%$, específicamente en la muestra de Portugal $(48,15 \%)^{7}$ y de China $(36,96 \%)^{12}$. En este estudio el valor del KMO fue superior a 0,70 , semejante a lo que paso en la muestra de China $(K M 0=0,72)^{12}$ y en la muestra de Nueva Zelanda $(\mathrm{KMO}=0,89)^{14}$.

Este estudio permitió confirmar que el IBP/SVG está ajustado a los datos. En la evaluación de la validad predictiva de los ítems/dimensiones para la medida de bien-estar subjetivo (IBP/SVG), la varianza explicada fue superior a $90 \%$. En el estudio portugués fue de $62 \%$, con varianza unitaria de $17 \%$ y varianza compartida de $45 \%^{7}$, en el estudio australiano a varianza unitaria fue de $8 \%$ y la compartida de $18,5 \%{ }^{10}$, en estudio tailandés la varianza explicada fue de $61,9 \%{ }^{13}$, en la muestra china la varianza explicada fue de $47 \%$, con varianza unitaria de $28 \%$ y compartida de $39 \%^{12}$. En Nueva Zelanda la varianza fue de $64 \%$, con varianza unitaria de $15 \%$ y $13 \%$ y varianza compartida de $49 \%$ y $52 \%{ }^{14}$. En este estudio los ítems que más contribuyen para el IBP son la salud y el nivel de vida, mientras en otros estudios, $11-14$ fue el nivel de vida y la seguridad. Estas diferencias pueden estar relacionadas con cuestiones culturales y con la propia situación de salud de las personas con ERC. En la validad convergente, tal como lo esperado se encontró soporte para la 
relación entre el BES (IBP/SVG) y las otras medidas de Bien-estar, específicamente la Felicidad Subjetiva (SHS) y el AP y AN (PANAS). En las personas con ERC, los valores altos de IBP/SVG están relacionados a valores altos de Felicidad Subjetiva y AP, y contrariamente, asociado al $\mathrm{AN}$. Un estudio español ${ }^{15}$ mostró asociación al soporte social, depresión y sentido de coherencia. EI IBP/SVG consigue discriminar diferencias culturales; las personas con ERC portuguesas tienen niveles más bajos de BES relativamente a las personas con nacionalidad de un país de África. También se encontraron diferencias en las muestras representativas de la población de Brasil, Colombia y Canadá. ${ }^{9}$

Estos resultados son semejantes a los de la escala original ${ }^{8}$ y la versión en portugués europe ${ }^{7}$, y sugieren que esta medida de BES es válida y confiable para evaluar la satisfacción con la vida en general, en personas con ERC.

Este estudio usó una muestra representativa de personas con ERC sometida a hemodiálisis.

En futuras pesquisas se recomienda la realización de un análisis factorial confirmatoria del IBP/SVG en esta población específica. Además se recomienda, a los enfermeros y investigadores en salud, la utilización de esta medida de BES en futuras investigaciones, una vez que esta medida traduce un indicador de salud importante para la calidad de vida en las personas con ERC ${ }^{16}$.

\section{Conclusiones}

La validación de la versión portuguesa del IBP/SVG tiene propiedades psicométricas adecuadas y ajustadas para las personas con ERC. En este estudio se encuentra soporte para la estructura uní-factorial de esta escala, y para la confiabilidad y estabilidad. EI IBP/SVG es una medida válida y reproductible para ser aplicada sobre la forma de cuestionario, o entrevista, en personas en situaciones de hemodiálisis, para evaluar el BES.

Recibido: 9 octubre 15

Revisado: 24 noviembre 15

Modificado: 11 enero 16

Aceptado: 18 enero 16

\section{Bibliografía}

1. Patat CL, Stumm EMF, Kirchner RM, Guido LA, Barbosa DA. Análisis de la cualidad de vida de usuarios en hemodiálisis. Enferm. Glob. 2012; 11(27):54-65.

2. Poveda VDB, Alves JDS, Santos ED. Diagnósticos de enfermagem en pacientes submetidos à hemodiálise. Enferm. Glob. 2014; 13(2):60-81.

3. Guerra-Guerrero V, Sanhueza-Alvarado 0 , Cáceres-Espina M. Quality of life in people with chronic hemodialysis: association with sociodemographic, medical-clinical and laboratory variables. Rev. Latino-Am. Enferm. 2012 set-out [Acesso 2015 ago 23]; 20(5):838-846.

4. Oller GASAI0, Ribeiro RCHM, Travagim DSA, Batista MA, Marques S, Kusumota L. Functional independence in patients with chronic kidney disease being treated with haemodialysis. Rev. Latino-Am. Enferm. 2012; 20 (6):1033-1040.

5. Pérez Domínguez, T., Rodríguez Pérez, A., Suárez Álamo, J., Rodríguez Castellano, L., García Bello, M. Á., \& Rodríguez Pérez, J. C. (2012). Satisfacción del paciente en una Unidad de Diálisis: ¿Qué factores modulan la satisfacción del paciente en diálisis? Enferm. Nefrol. 2012; 15(2), 101-107.

6. Forjaz MJ, Prieto-Flores $M E$, Ayala $A$, RodríguezBlázquez C, Fernández-Mayoralas G, Rojo-Perez F, Martínez-Martín P. Measurement properties of the Community Wellbeing Index in older adults. Qual. Life Res. 2011; 20(5):733-743.

7. Ribeiro JP, Cummins R. 0 bem-estar pessoal: estudo de validação da versão portuguesa da escala. In Actas do $7^{\circ}$ Congresso Nacional de Psicologia da Saúde. Lisboa: ISPA. 2008: 5058. Disponible en: http://repositorio-aberto.up.pt/ handle/10216/21065

8. Cummins RA, McCabe MP, Romeo Y, Gullone E. The Comprehensive Quality of Life Scale: Instrument development and psychometric evaluation on tertiary staff and students. Educ. Psychol. Measur. $1994 ;$ 54: 372-382. 
9. Wills-Herrera E, Islam G, Hamilton M. Subjective well-being in cities: A multidimensional concept of individual, social and cultural variables.

Appl. Res. Qual. Life. 2009; 4(2): 201-221

10. McGillivray JA, Lau ALD, Cummins RA, Davey $G$. The utility of the personal wellbeing index intellectual disability scale in an Australian sample. J. Appl. Res. Intellect. Disabil. 2019; 22(3): 276-286.

11. Nielsen I, Smyth R, ZhaiQ. Subjective wellbeing of China's off-farm migrants. J. Happiness Stud.2010; 11(3): 315-333.

12. Nielsen I, Paritski 0, Smyth R. Subjective wellbeing of Beijing taxi drivers. J. happiness stud. 2010; 11(6): 721-733.

13. Yiengprugsawan $V$, Seubsman $S A$, Khamman $S$, Lim LY, Sleigh AC. Personal Wellbeing Index in a national cohort of 87,134 Thai adults. Soc. Ind. Res. 2010; 98(2): 201-215.

14. Ganglmair-Wooliscroft A, Lawson R. Applying the international wellbeing index to investigate subjective wellbeing of New Zealanders with European and with Maori heritage. Kotuitui: New Zealand j. Soc. Sci. Online. 2008; 3(1): 57-72.

15. Forjaz MJ, Ayala A, Rodríguez-Blázquez C, Prieto-Flores ME, Fernandez-Mayoralas G, Rojo-Pérez F, Martínez-Martín P. Rasch analysis of the International Wellbeing Index in older adults. Int. psychogeriatr. 2012; 24(02): 324-332.

16. Bennett PN, Weinberg MK, Bridgman T, Cummins RA. The happiness and subjective well-being of people on haemodialysis. J. Ren. Care. 2015; 41(3): 156-161.

17. Galinha I, Ribeiro J. Contribuição para 0 estudo da versão portuguesa da Positive and Negative Affect Schedule (PANAS): II - Estudo psicométrico. Anal Psicol. 2005; 23(2):219-227. Disponible en: http://publicacoes.ispa.pt/index. php/ap/article/viewFile/84/pdf

18. 18. Pais-Ribeiro JL. Validação transcultural da Escala de Felicidade Subjectiva de Lyubomirsky e Lepper. Psicol. Saúde Doenças. [Internet]. 2012[Acesso 2015 ago 19]; 13(2): 157-168.
Disponível em: http://www.scielo.mec.pt/pdf/psd/ v13n2/v13n2a03.pdf

19. De Lima DVM. Desenhos de pesquisa: uma contribuição para autores. Online Braz J Nurs. (Online). 2011; 10(2). Disponible en: http:// www.objnursing.uff.br/index.php/nursing/article/ view/3648/html

20. Santos GEO. Cálculo amostral: calculadora online Disponible en: <http://www.calculoamostral. vai.la>.

21. Sousa LMM, Marques-Vieira CMA, Carvalho ML, Veludo $F$, José, HMG. Fidelidade e validade na construção e adequação de instrumentos de medida. Enformação. 2015; 5:25-32. Disponible en: http:// www.acenfermeiros.pt/index.php?idl=15\&id2=9 